\title{
Lidil
}

Revue de linguistique et de didactique des langues

$50 \mid 2014$

Variation stylistique et diversité des contextes de socialisation

\section{Le français, « langue maternelle » est-il une « langue vivante »? Réflexion sur la place de la variation stylistique dans le discours scolaire}

Is French Language "as a Mother Tongue" a "Living" Language? Reflection on

the Role of Stylistic Variation in the Educational Discourse

\section{Emmanuelle Guerin}

\section{OpenEdition}

Journals

Édition électronique

URL : http://journals.openedition.org/lidil/3609

DOI : 10.4000/lidil.3609

ISSN : 1960-6052

\section{Éditeur}

UGA Éditions/Université Grenoble Alpes

\section{Édition imprimée}

Date de publication : 15 décembre 2014

Pagination : 147-167

ISBN : 978-2-84310-287-5

ISSN : $1146-6480$

\section{Référence électronique}

Emmanuelle Guerin, « Le français, « langue maternelle » est-il une « langue vivante » ? Réflexion sur la place de la variation stylistique dans le discours scolaire », Lidil [En ligne], 50 | 2014, mis en ligne le 15 juin 2016, consulté le 19 avril 2019. URL : http://journals.openedition.org/lidil/3609; DOI : 10.4000/ lidil.3609 


\title{
Le français, «langue maternelle» est-il une "langue vivante »? Réflexion sur la place de la variation stylistique dans le discours scolaire
}

\author{
Emmanuelle Guerin*
}

\begin{abstract}
RÉSUMÉ
Dans cet article, je m'attache à interroger la place de la variation stylistique dans l'enseignement du français, langue maternelle. Considérant que cette dimension de la variation est le signe de la «vivacité» d'une langue, comment est-elle abordée lorsqu'il s'agit d'enseigner des savoirs relatifs à la propre langue des locuteurs concernés? Pour répondre à cette question, c'est la notion même de «langue maternelle » qu'il convient de caractériser puisque, au moins dans le cas du français, elle renvoie à un ensemble circonscrit de formes et de pratiques qui ne sont pas celles observables dans le quotidien extra-scolaire des élèves. Compte tenu des effets de l'idéologie du standard, on pose l'hypothèse d'une déconsidération de la variation stylistique, pour ce qu'elle est effectivement, dans le discours scolaire, au profit d'une représentation hiérarchisée et idéalisée, qui serait partie prenante des difficultés rencontrées par les élèves.
\end{abstract}

\section{ABSTRACT}

In this paper, I will question the role of stylistic variation in the teaching of French as a mother tongue. Since variation is supposed to be a sign of the "vitality" of a language, how is this point addressed when teaching knowledge about speakers' own language? To answer this question, the concept of "mother tongue" has to be discussed, since, at least as far as French language is concerned, it suggests a restricted setting of forms and practices which is different from what can be observed in ordinary language outside school. Given the impact of standard ideology on teaching practice, I hypothesize that stylistic variation is discredited in favor of hierarchical and idealized representation, which can be considered as one of the causes of pupils'difficulties.

* Université d'Orléans, Laboratoire ligérien de linguistique (UMR 7270). 


\section{Introduction}

Partant du principe que la variation stylistique est un trait définitoire des langues et de leur dynamique, qu'il en va de la «santé» d'une langue (Gadet \& Tyne, 2007), l'enseignement d'une langue intègre, d'une manière ou d'une autre, ses effets. Dans cet article, il s'agit de regarder comment se manifeste cette intégration, et quels en sont les enjeux et répercussions sur la description proposée aux élèves/apprenants. En particulier, ce texte s'intéresse à l'enseignement de la «langue maternelle» en France, entendons l'enseignement de la langue dispensé à des enfants, locuteurs du français. Il a été montré que la compétence nécessaire à la variation stylistique s'acquiert dans l'enfance, naturellement, par l'expérience quotidienne d'interactions de plus en plus variées (voir par exemple, Andersen, 1990). Partant, comment l'enseignement de la «langue maternelle» accompagne-t-il les élèves dans le développement et l'enrichissement de cette compétence? La reconnaissance partagée par l'ensemble de la communauté d'un «bon usage» du français (par exemple : Charmeux, 1989 ou encore Ledegen, 2000) exclusif a-t-elle un effet sur cet enseignement?

Tenter de répondre à ces questions passe par la problématisation de la notion de «langue maternelle» qui laisse apparaitre toute sa complexité, notamment parce que, comme le soulignent Gueunier et Genouvrier (1982, p. 5),

si le linguiste ne peut traiter exhaustivement de la langue maternelle, il ne peut non plus faire l'économie d'y penser, en faisant appel à d'autres disciplines des sciences humaines.

Ainsi, la définition de la «langue maternelle» n'est pas évidente. En fonction des lieux où il est pertinent de la considérer, elle se teinte de présupposés théoriques qui ne sont pas du même ordre, voire qui peuvent s'opposer d'une définition à l'autre. Si, pour le linguiste, il n'y a pas de caractérisation exhaustive de la «langue maternelle», les enjeux sociaux de son enseignement appellent l'arrêt d'un modèle unique et commun ${ }^{1}$, ce qui conduit, dans ce cadre, à l'hypothèse de l'opposi-

1. Chiss et David (2012, p. 138) affirment qu'une vision pertinente de la réalité linguistique «peut conduire à une antinomie didactique et pédagogique (quelle forme de stabilisation du savoir à transmettre?) tout en reposant le problème épistémologique chomskyen - et néanmoins incontournable de la prédictibilité». 
tion «langue maternelle»/«langue vivante». Ceci posé, quelle place pour la variation en général et stylistique en particulier, dans la description scolaire de la langue?

Je tente de montrer, dans cet article, les implications de cette opposition terminologique dans les pratiques d'enseignement, en France. L'observation des programmes et manuels scolaires, autrement dit l'aspect institutionnel du discours scolaire, révèle une différence d'approche de la langue, que l'objet d'enseignement intègre ou non la catégorie des «langues vivantes». En l'occurrence, lorsque ce n'est pas le cas, lorsque l'on a affaire à la «langue maternelle», la variation est codifiée, pour donner à voir un usage idéalisé de la langue, conformément au principe sur lequel repose l'idéologie du standard (Milroy \& Milroy, 1985).

\section{L'enseignement du français langue maternelle, langue des élèves?}

«La France championne des inégalités scolaires», titrait Le Monde.fr, daté du 3 décembre 2013, à la suite de l'annonce de la mauvaise note attribuée par l'OCDE à la France, dans le cadre de son Programme international pour le suivi des acquis des élèves (PISA). En effet, bien que l'on puisse certainement reprocher le manque de nuance de l'approche quantitative de ce type de programme ${ }^{2}$, l'observation du monde scolaire permet de confirmer que les écarts se creusent entre les élèves issus de milieux dits «favorisés» et ceux issus de milieux dits «défavorisés » ${ }^{3}$. Parmi les hypothèses avancées pour expliquer cet écart, deux sont généralement privilégiées, pour ce qui concerne l'enseignement de la discipline «français » : l'une qui met en rapport la réussite/l'échec des

2. Peut-être, dans une approche cette fois qualitative, aurait-on intérêt à accorder au moins autant d'importance au phénomène de «résilience scolaire», tel que décrit notamment par Bouteyre (2004). Certes, la réussite des enfants issus de milieux dits défavorisés est bien moins saillante, mais n'y trouverait-on pas des pistes de réflexion intéressantes? De la même façon, il ne faudrait pas négliger l'échec rencontré par les élèves issus de milieux dits favorisés, les «héritiers», pour reprendre Henri-Panabière (2010) s'inspirant du concept bourdieusien.

3. «Favorisés » / « défavorisé » sont des termes qui décomplexifient les situations en les caricaturant (voir Gadet, 2003). 
élèves et la place de l'écrit dans leur environnement familial (Lahire, 1993), et l'autre qui incrimine le «bi- ou multi-linguisme» des élèves ${ }^{4}$.

Concernant la première hypothèse, nul doute que la familiarité avec l'écrit (un certain type d'écrits), dans le cadre familial, favorise les apprentissages quand on sait ce qui est effectivement enseigné (Guerin, 2008). Cependant, compte tenu de l'évolution des pratiques, n'auraiton pas intérêt à revoir ce qui est entendu par «écrit»? Manifestement, dans tous les milieux, l'écrit est plus que jamais présent dans l'univers des élèves, étant donné leur usage d'Internet et du SMS. Les productions émergeant de ces médias ne sont pas considérées comme des écrits légitimes par l'institution scolaire, voire la société dans son ensemble. Si les linguistes, notamment à la suite des travaux de Jacques Anis en France, y voient des formes qui s'inscrivent dans la continuité de l'évolution des pratiques d'écriture (Gadet, 2008), il subsiste dans l'opinion publique une certaine réticence à l'égard du phénomène perçu comme un «désastre discursif et scriptural pour les plus pessimistes», comme le soulignent Fairon et Klein (2010, p. 113). Selon David et Goncalves (2007), cette résistance résulte du «fait que l'écriture texto brouille les frontières entre le français écrit et oral, au point d'assimiler le premier à de l'oral conversationnel» (p. 46). Ce jugement de valeur est très tôt partagé par les élèves eux-mêmes qui, imprégnés de l'idéologie scolaire concourant à la survalorisation d'un certain écrit (par exemple, Boutet, 2002), excluent de la pratique scripturale des productions qui leur sont pourtant familières. Lors d'entretiens menés dans une école primaire d'un quartier «sensible» de la ville de Montargis, une élève de CM2 me dit qu'en dehors de l'école, jamais elle n'écrit ni ne lit. Or, plus tard dans la conversation, elle m'explique qu'elle fréquente quotidiennement le réseau social Facebook. Je lui fais alors remarquer qu'elle est donc régulièrement en contact avec l'écrit, ce à quoi elle répond qu'elle ignorait que c'était de l'écrit. Cette anecdote, si elle peut prêter à sourire, illustre, du moins il me semble, ce qui pourrait être à l'origine de certaines difficultés rencontrées par les élèves. On comprend par la réponse de cette élève de CM2 que, pour elle, il n'est

4. La question du bi- ou plurilinguisme des élèves pourraient à elle seule faire l'objet d'un article tant il n'est pas évident de déterminer ce qu'est effectivement un bi- ou plurilingue. Par ailleurs, on attribue souvent cette étiquette à des enfants parce que les parents sont locuteurs d'une langue étrangère, sans réellement se poser la question du rapport que les enfants entretiennent avec cette langue. 
pas envisageable qu'une production écrite puisse ne pas se conformer aux règles telles qu'elles sont enseignées à l'école. Autrement dit, il n'y aurait pas de variation possible à l'écrit. Cette représentation se construit notamment avec ce que donne à voir le discours scolaire, comme par exemple dans cet extrait d'un manuel destiné aux élèves de CE2 :

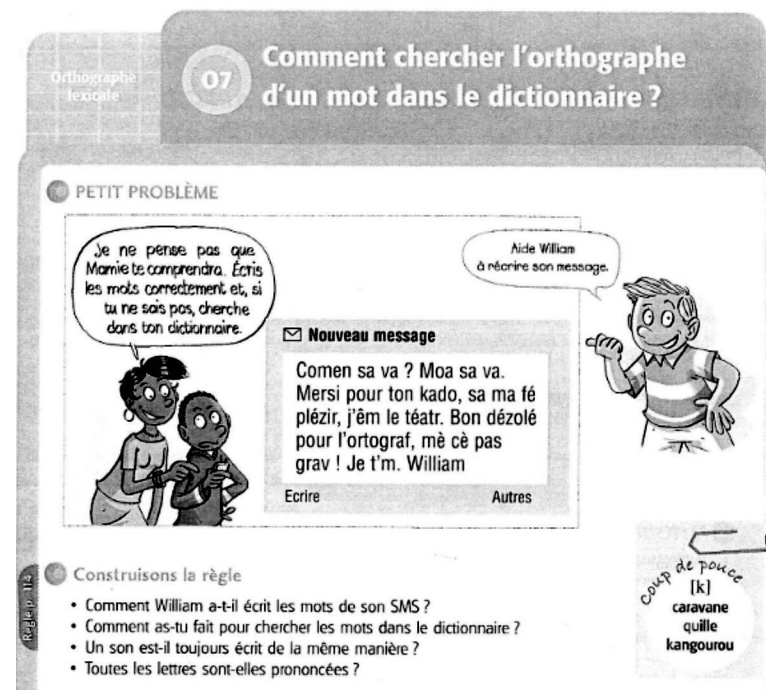

Extrait 1. - Interlignes CE2, CED éditions, p. 112.

Bien que, dans un premier temps, l'explication de la mère de William introduise l'idée de variation en alertant ce dernier sur la nécessité de s'adapter à son lecteur, la piste est immédiatement abandonnée puisqu'il n'est en fait pas question d'adaptation mais de conformité à la forme «correcte» («Écris les mots correctement»). On voit ainsi que le rapport aux écrits s'inscrit dans une logique d'exclusivité. Pourtant, de récentes études ont montré qu'il n'y aurait pas de corrélation directe entre la maitrise de différents codes graphiques et celle du code standard, voire l'une et l'autre pourraient s'alimenter (par exemple : Bouillaud et coll., 2007 ou encore Fairon \& Klein, 2010). Ainsi, on peut se demander si un positionnement différent de l'institution face à l'écrit, c'est-à-dire l'acceptation de l'existence de la variation de l'écrit, ne pourrait pas faciliter l'enseignement de la forme légitime.

La seconde hypothèse que nous avons évoquée soutient le rapport entre les difficultés rencontrées par les élèves et leur supposé bi- ou 
multi-linguisme. Là encore, on ne peut pas ignorer qu'un environnement linguistique familial où les élèves sont quotidiennement impliqués dans des échanges mettant en jeu la langue (la forme de langue) enseignée à l'école favorise les apprentissages. Cependant, ceci ne vaut que parce que l'enseignement de la discipline «français» se confond avec la transmission de savoirs "clé en main», sans qu'on ne fasse appel à la réflexivité des élèves, c'est-à-dire à leur capacité à construire eux-mêmes les savoirs par l'expérimentation. Les textes officiels ne présentent pas les choses en ces termes, puisque d'une part, on y pose d'emblée la multiplicité des langues étrangères familiales comme un «handicap» (Guerin, 2013) tout en vantant, d'autre part, les mérites de l'apprentissage précoce de langues étrangères, notamment pour favoriser la compétence métalinguistique des élèves. Autrement dit, la maitrise de plusieurs langues est envisagée comme un atout, tant que celles-ci entrent dans la liste fermée des langues enseignées, qui n'intègre pas les langues de l'immigration, pourtant les plus courantes en France. C'est ainsi que les bilinguismes arabe-français, wolof-français, etc., ne sont pas reconnus comme des valeurs ajoutées, à l'image d'un apprentissage précoce de l'anglais ou de l'allemand, mais comme des freins dans les apprentissages relatifs à la discipline «français» (Billiez, 2007). Par ailleurs, pourquoi considérer que la multiplicité des langues dans les usages quotidiens serait davantage problématique pour les élèves concernés que la multiplicité inévitable des formes d'une même langue, en l'occurrence le français? Comme le souligne Mougeon (1998) s'appuyant sur les études portant sur le code-switching (en particulier, Heller, 1988) :

En effet, dans les communautés où plusieurs langues ou dialectes sont en contact, ces variétés remplissent les mêmes fonctions que différentes variétés d'une même langue dans une communauté unilingue. Elles ont donc une fonction stylistique. (p. 70)

Dès lors, les questions qui se posent à propos des difficultés rencontrées par les élèves issus de milieux plurilingues devraient se poser de la même façon pour les autres élèves ou ne pas se poser du tout.

Dans les deux cas, on peut constater qu'il s'agit toujours de poser le problème en y cherchant des raisons du point de vue des élèves et de ce qu'ils auraient en moins ou en plus. Or, compte tenu de ce qui vient d'être dit du rapport à l'écrit, qui pourrait être repensé en d'autres termes, ou de la présence de langues étrangères dans l'environnement familial, qui pourrait ne pas être si éloignée de celle de différentes 
formes d'une même langue, la pertinence du point de vue peut être remise en question. Pourquoi ne pas se demander si l'écart entre les élèves ${ }^{5}$ ne pourrait pas être un construit de l'institution scolaire? L'un des leviers de cette construction serait le rapport que les enfants entretiennent avec leur «langue maternelle», telle qu'elle leur est présentée dans le discours scolaire.

\section{3. "Langue maternelle» : une notion complexe}

L'expression «langue maternelle» (LM) sert à distinguer la langue dans laquelle les individus sont, à priori, socialisés, par opposition aux langues qui peuvent être apprises secondairement. Cependant, dans les faits, tout n'est pas si évident et ce à quoi elle renvoie est bien plus complexe qui n'y parait. Herlitz et coll. (2007) envisagent une caractérisation de l'objet qui renvoie à trois réalités :

- la home language, la langue des premiers échanges, développée dès l'enfance, avant les apprentissages scolaires;

- la langue du fatherland, qui s'inscrit à un niveau politique et culturel (par opposition à la première acception qui s'inscrit à un niveau individuel, même si les deux niveaux sont nécessairement imbriqués), qui conditionne l'identité régionale ou nationale;

- la langue en tant qu'objet de l'enseignement destiné à ses locuteurs, qui se confond avec la forme standard.

Si les deux derniers aspects de la notion peuvent, au moins dans la plupart des pays d'Europe de l'Ouest, concerner la même forme de la langue, ce que recouvre le premier aspect renvoie à des formes hétérogènes, imprévisibles, qui ont en commun un écart plus ou moins grand avec la forme standard. Pourtant, si, dans tous les cas, on parle de LM c'est qu'il est toujours question de la langue dans laquelle évoluent et se construisent les locuteurs dont l'identité est à la fois individuelle et collective. Penser une LM revient donc à penser l'imbrication des trois aspects de la notion, observable sous deux angles :

5. Évidemment, il ne s'agit pas de nier les différences sociales. C'est pourquoi je parle d'écart entre les élèves, exclusivement considérés en tant que récepteurs du discours scolaire, et non entre les enfants. 
- du point de vue de l'individu (premier aspect), elle se joue sur le plan des représentations. Même si la home language n'est jamais la forme standard, celle-ci n'est jamais ignorée. Elle influence plus ou moins fortement, par imitation ou par opposition, selon les cas, les pratiques quotidiennes;

- en revanche, du point de vue du collectif (deux derniers aspects), les effets de l'imbrication ne relèvent pas de l'influence de la home language. La conceptualisation de la forme standard n'intègre les pratiques individuelles qu'au titre de contre-exemples, puisque, par définition, le principe de standardisation s'oppose au principe de variation.

The 'standard language' interpretation of mother tongue furthermore disregards the many regional and local varieties of that standard, it disregards the multilingual construction of nowadays society. (Herlitz et coll., 2007, p. 16)

Comme le souligne Halliday (2007, p. 28), l'enseignement de la LM, dans sa fonction prescriptive, n'ajoute rien à la performance de l'élève mais la rend plus socialement acceptable.

Ceci étant dit, ce n'est pas la diversité des pratiques quotidiennes des locuteurs d'une langue qui apparait comme la principale caractéristique d'une LM. Les formes non standard sont davantage perçues comme des dommages collatéraux. Une LM est principalement considérée relativement à sa forme légitime.

\section{4. «Langue maternelle / « langue vivante»}

Partant, la possibilité d'envisager qu'une LM puisse être une langue vivante est remise en question. Bien qu'on sache dire ce qu'est une langue vivante, lorsqu'il s'agit d'en donner un exemple, elle peut prendre un caractère restrictif, notamment pour les locuteurs du français qui ont encore en tête la terminologie scolaire. Elle a une double référence : l'une implicite, partagée, qui active le sens de «vivante» et l'autre explicite qui donne à l'adjectif un sens secondaire, celui suggéré par la tradition scolaire. Dans les textes officiels, on ne reconnait que les langues vivantes étrangères. Le français n'est ainsi nommé qu'à la condition qu'il soit enseigné à un public non francophone (FLE, FLS, FOS, FLP, FLSco...). Ceci explique que, dans le cadre de l'enseignement du français LM, on ne trouve pas mention de son caractère «vivant» dans les textes officiels. Vigner (2003, p. 156-157) explique, après s'être étonné 
de l'absence du français dans l'ensemble des «langues vivantes », que l'expression ne serait qu'un reliquat de l'opposition langue vivante/ langue morte, la seconde catégorie n'étant plus en usage. Cet argument n'est pas satisfaisant car lorsque l'opposition n'était pas contestée par les hellénistes et les latinistes, le français n'était pas davantage considéré comme une langue vivante dans les discours institutionnels. Cette catégorisation des langues n'est pas sans conséquence sur leur enseignement. L'enseignement d'une langue vivante est, à ce titre, soumis à un certain nombre de contraintes qui ne semblent pas affecter l'enseignement du français LM.

En pratique, «maternelle» et «vivante» s'opposent. Si les deux adjectifs ne sont pas, dans leur sens premier, antagonistes, l'usage qui en est fait dans le discours institutionnel met en lumière deux domaines de référence contradictoires. L'un, «vivante», suppose le dynamisme, l'autre, «maternelle», une certaine stabilité puisqu'il implique la remise en jeu perpétuelle d'une langue telle qu'observable dans les pratiques de la «mère ${ }^{6} »$. Il y a l'idée de la transmission d'un héritage de génération en génération. En revanche, la pratique contemporaine de l'enseignement d'une langue vivante répond, depuis la rénovation de l'enseignement des langues vivantes (B.O. $\mathrm{n}^{\circ} 23$ du 8 juin 2006), aux principes énoncés par le Cadre européen commun de référence pour les langues (CECRL). Il y est clairement fait mention de l'importance de l'intégration au répertoire linguistique des élèves/apprenants des savoirs relatifs à la nouvelle langue. La langue y est pensée comme réappropriable, donc évolutive :

Il ne s'agit plus simplement d'acquérir la «maitrise» d'une, deux, voire même trois langues, chacune de son côté, avec le «locuteur natif idéal» comme ultime modèle. Le but est de développer un répertoire langagier dans lequel toutes les capacités linguistiques trouvent leur place. [...] En outre, une fois admis le fait que l'apprentissage d'une langue est le travail de toute une vie, le développement de la motivation, de la capacité et de la confiance à affronter une nouvelle expérience langagière hors du milieu scolaire devient primordial. (CECRL, p. 11)

6. «Mère» ne fait pas ici intervenir nécessairement l'idée de filiation, mais plutôt celle d'antécédent au sens large. «Langue maternelle» équivaut en fait à «langue nationale», «langue de la patrie». Ce sont les contextes d'apparition qui motivent l'emploi de l'une ou l'autre : la première expression sert à faire la distinction avec le français langue étrangère, plutôt dans un contexte scolaire. La seconde s'inscrit généralement dans les discours plus politiques. Dans ce cas, la distinction avec le FLE n'est plus pertinente. 
De cet extrait du texte, on peut relever un certain nombre d'éléments qui explicitent les contraintes liées à l'enseignement d'une langue vivante. On peut s'arrêter premièrement sur «maitrise», mis entre guillemets. Ces derniers supposent qu'on ne saurait envisager la maitrise totale et absolue d'une langue puisqu'il est précisé que «l'apprentissage d'une langue est le travail de toute une vie». Il semble que «langue» ici n'implique pas la LM puisque, dans les programmes scolaires de français, on parle de la maitrise de la langue comme une priorité de l'école élémentaire, cette fois sans guillemets. Cette remarque n'est pas anecdotique car elle suggère en substance qu'on serait en mesure de stabiliser et de transmettre tous les savoirs sur une langue, niant de fait son caractère vivant. Dès lors, la notion de «locuteur natif idéal», rejetée par les principes du CECRL, intègre le discours scolaire. Par ailleurs, la mise en avant d'un «répertoire langagier dans lequel toutes les capacités linguistiques trouvent leur place» permet également d'appuyer la distinction LM/langue vivante. Pour l'enseignement de cette dernière, on propose une représentation horizontale des compétences, là où, en LM, elles s'organisent selon un axe vertical, une hiérarchie au sommet de laquelle on trouve les compétences visées. On peut ainsi lire, à propos du travail sur le langage oral, dans le B.O. hors série $\mathrm{n}^{\circ} 3$ du 19 juin 2008 :

Dans des situations d'échanges variées, il [l'élève] apprend à tenir compte des points de vue des autres, à utiliser un vocabulaire précis appartenant au niveau de la langue courante, à adapter ses propos en fonction de ses interlocuteurs et de ses objectifs.

On est loin ici d'un «répertoire langagier dans lequel toutes les capacités linguistiques trouvent leur place» puisqu'on comprend que l'objectif de l'apprentissage est une uniformisation de ces capacités pour que les élèves soient en mesure de produire exclusivement de la «langue courante», bien que, et de façon contradictoire, on reconnaisse par ailleurs que les élèves sont impliqués «dans des situations d'échanges variées». Compte tenu de cette remarque, on peut s'interroger sur le sens à donner à «adapter» puisqu' apparemment, la maitrise de la langue semble se confondre avec la maitrise d'une unique forme, la «langue courante». 


\section{La variation (stylistique) dans l'enseignement de la langue « maternelle» « vivante»}

Étant donné les implications des dénominations, constate-t-on une différence d'approche de la langue lorsque l'enseignement est destiné à un public d'apprenants non francophones? Les enjeux ne sont nécessairement pas les mêmes que lorsqu'il s'agit de la LM. Conséquemment, on n'approche pas l'objet enseigné selon les mêmes méthodes. Néanmoins, dans les deux cas, la langue est la même, les unités qui la constituent sont les mêmes, son ancrage dans la culture de la communauté de ses usagers est le même. Des différences ne devraient pas apparaitre au-delà de la façon d'aborder les savoirs, de l'«enrobage », qui s'adapte aux élèves/apprenants. Or, on constate un décalage notable dans la description même de la langue. Cela est principalement dû au positionnement que l'on peut qualifier d'idéologique par rapport à la langue. Lorsque le français est une langue étrangère, son enseignement est celui d'une langue vivante; on tente de rendre compte d'une certaine réalité ${ }^{7}$ des usages. Inversement, en français LM, l'enseignement tente de régler les usages des élèves. Ces deux logiques s'illustrent particulièrement bien dans les textes officiels, comme on a pu le voir, mais aussi dans les outils pédagogiques. Par exemple, on peut lire dans un manuel de français à l'usage de la communauté chinoise que le pronom (ici, pronom indéfini) «on» équivaut à «nous» dans la langue courante.

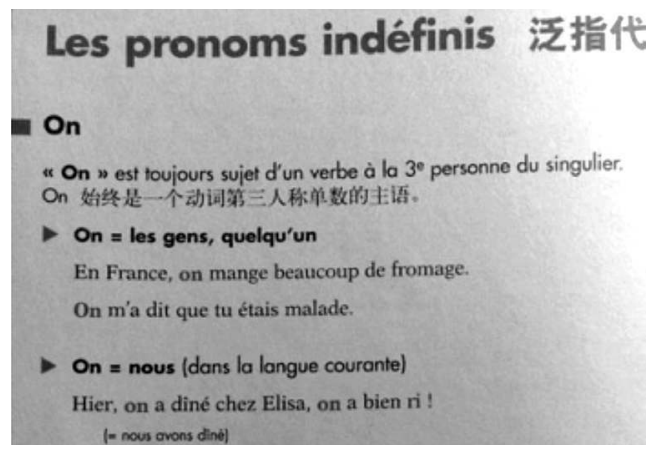

Extrait 2. - Grammaire pratique du français (version chinoise), Hachette, 2000 (réimprimé en 2007).

7. Le français enseigné n'est pas, là non plus, à l'image des usages réels. Néanmoins, beaucoup de travaux s'interrogent sur la pertinence et la façon de tendre à davantage de réalisme dans l'enseignement du français langue étrangère (voir par exemple le numéro 31 de la revue Mélanges). 
Si l'on considère que «langue courante» renvoie aux pratiques quotidiennes majoritaires des locuteurs français, on peut effectivement dire que ce manuel illustre la démarche qui part des usages observables pour proposer une description qui tend à une représentativité de la réalité, même si l'équivalence n'est en fait pas tenable et que la maintenir entretient une représentation faussée de la langue. Cependant, c'est le positionnement du discours par rapport à la variation de la langue qui nous intéresse ici. La logique inverse est illustrée dans l'extrait d'un manuel de grammaire à destination des classes de CM1, conforme aux programmes de 2008 :

\section{Les niveaux de langue}

- Selon les circonstances dans lesquelles on s'exprime, on utilise un niveau de langue différent : familier, courant ou soutenu.

- Le niveau de langue se remarque d'abord par le vocabulaire employé.

- La plupart du temps, dans la vie quotidienne, on utilise le langage courant :

Nous ne pouvons pas changer de voiture maintenant.

- Avec ceux que l'on connaît bien (les amis, les proches...), on peut utiliser

le langage familier :

On peut pas changer de bagnole maintenant. $\rightarrow$ oublie de ne, emploi du mot bagnole

- À l'écrit ou avec des gens que l'on ne connaît pas bien, on utilise le langage

soutenu :

Il ne nous est pas possible de changer de véhicule pour le moment.

Extrait 3. - Demongin Christian (dir.), Français CM1. Mille-feuilles,

Paris, Nathan, 2012.

Ici, on apprend que «on» n'illustre pas le «langage courant», comme précédemment, mais le «langage familier». Il s'agit donc de convaincre des enfants de neuf ans que, «dans la vie quotidienne», la communauté des locuteurs français, dont ils font partie, utilise principalement «nous», en lieu et place de «on» qu'elle réserve aux interlocuteurs intimes qui, à en croire le découpage en trois niveaux ${ }^{8}$, ne participent pas de ladite vie quotidienne. Plus troublant encore, dans le manuel, destiné aux CM2 ${ }^{9}$, «on» a totalement disparu de la description. Aucune mention au chapitre des pronoms personnels et pas davantage à celui des pronoms indéfinis, catégories traditionnellement retenues pour classer un inclassable. On peut en déduire que, dans le cadre du français

8. Pour une problématisation des «niveaux de langue», tels qu'ils sont présentés par la grammaire scolaire, voir Paveau (2008).

9. C. Demongin (dir.), Français CM2. Mille-feuilles, Paris, Nathan, 2013. 
LM, l'usage reconstruit, idéalisé, des locuteurs (donc des élèves euxmêmes) se caractérise par un emploi de «on» qui n'est pas courant au point qu'il n'est plus utile d'en parler à partir d'un certain niveau supposé de «maitrise de la langue». Cet exemple illustre l'opposition langue vivante/LM qui met en lumière des positions contradictoires.

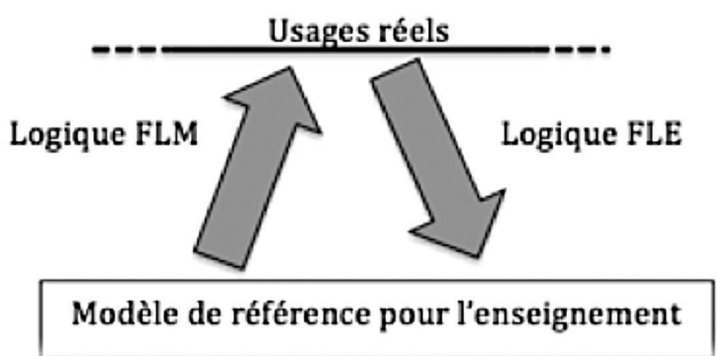

Logiques contradictoires en FLM et FLE.

Pour maintenir cette opposition, on n'hésite pas à proposer une description de la langue, à mon sens, largement absurde : affirmer que «on» n'est pas courant relève de l'incongruité lorsque l'on s'adresse à un public d'élèves français.

Dans le cadre du français LM, les questions relatives aux compétences langagières, autres que celles strictement linguistiques (sociolinguistique et pragmatique) ne sont pas considérées pour ce qu'elles sont effectivement. On pourrait penser que ces compétences peuvent s'acquérir et se développer sans un enseignement particulier et que c'est pour cette raison qu'elles ne sont pas davantage travaillées. Ce serait un moindre mal. Ce n'est pas ainsi que les choses se passent : plutôt que de simplement les ignorer, ces compétences sont strictement encadrées et réduites à un ensemble de savoirs limité et ne font écho à rien d'observable, dans le quotidien de la grande majorité des enfants, en dehors des activités scolaires. En revanche, en langue étrangère, ces compétences occupent une place centrale dans la réflexion didactique. Ainsi, le CECRL prévoit, par exemple, une grille de «correction sociolinguistique» présentant les attitudes à atteindre pour chacun des niveaux d'apprentissage. Il y a bien l'idée que la compétence linguistique n'est pas dissociable des autres compétences, notamment ici, la compétence sociolinguistique : 


\section{CORRECTION SOCIOUNGUISTIOUE}

\begin{tabular}{|c|c|}
\hline \multirow[t]{2}{*}{$\mathbf{C 2}$} & $\begin{array}{l}\text { Manifeste une bonne maitrise des expressions idiomatiques et dialectales avec la conscience des niveoux connotatifs de } \\
\text { sens. } \\
\text { Apprécie complètement les implications sociolinguistiques et socioculturelles de la langue utilisée par les locuteurs natifs } \\
\text { et peut réagir en conséquence. }\end{array}$ \\
\hline & $\begin{array}{l}\text { Peut jouer efficocement le rôle de médiateur entre des locuteurs de la langue cible et de celle de sa communauté } \\
\text { d'origine en tenant compte des différences socioculturelles et sociolinguistiques. }\end{array}$ \\
\hline \multirow[t]{3}{*}{$\mathbf{C l}$} & $\begin{array}{l}\text { Peut feconnaitre un large éventail d'expressions idiomatiques et dialectales et apprécier les changements de registres: } \\
\text { peut devoir toutefois confirmer tel ou tel détoil, en particulier si l'occent n'est pos familier. }\end{array}$ \\
\hline & Peut suivre des films utilisant largement l'argot et des expressions idiomatiques. \\
\hline & $\begin{array}{l}\text { Peut utiliser la langue avec efficacité et souplesse dans des relations sociales, y compris pour un usoge affectif, allusif ou } \\
\text { pour plaisanter. }\end{array}$ \\
\hline \multirow[t]{3}{*}{ B2 } & $\begin{array}{l}\text { Peut s'exprimer avec assurance, clairement ef poliment dans un registre formel ou informel approprié ò la situotion et } \\
\text { aux personnes en cause. }\end{array}$ \\
\hline & $\begin{array}{l}\text { Pout poursuivre une relation suivie avec des locutours natifs sans les amuser ou les irriter sans lo vouloir ou los mettre en } \\
\text { situation de se comporter autrement qu'avec un locuteur natif. }\end{array}$ \\
\hline & Peut s'exprimer convenablement en situation et éviter de grossières erreurs de formulation. \\
\hline \multirow[t]{3}{*}{ B1 } & $\begin{array}{l}\text { Peut s'exprimer et répondre d̀ un large éventail de fonctions langogières en ufilisant leurs expressions les plus courantes } \\
\text { dans un registre neutre. }\end{array}$ \\
\hline & Est conscient des règles de politesse importantes et se conduit de manière oppropriée. \\
\hline & $\begin{array}{l}\text { Est canscient des différences les plus significatives entre les coutumes, les usages, les attitudes, les voleurs et les } \\
\text { croyances qui prévalent dans la communauté concernée et celles de sa propre communauté et en recherche les indices. }\end{array}$ \\
\hline \multirow[t]{3}{*}{ A2 } & $\begin{array}{l}\text { Peut s'exprimer et répondre oux fonctions langogières de bose telles que l'échange d'information et lo demande et } \\
\text { exprimer simplement une idée et une opinion. }\end{array}$ \\
\hline & $\begin{array}{l}\text { Peut entrer dans des relations sociales simplement mais efficacement en utilisant les expressions courantes les plus } \\
\text { simples et en suivant les usages de base. }\end{array}$ \\
\hline & $\begin{array}{l}\text { Peut se débrouiller dans des échanges sociaux très courts, en utilisant les formes quotidiennes polies d'accueil et de } \\
\text { contact. Peut faire des invitations, des excuses et y répondre. }\end{array}$ \\
\hline Al & $\begin{array}{l}\text { Peut établir un contact social de base en utilisant les formes de politesse les plus élémentaires; accueil et prise de congé, } \\
\text { présentations et dire « merci } \$, s^{\prime} \text { il vous plaît } \$ \text { excusez-moi } » \text {, etc. }\end{array}$ \\
\hline
\end{tabular}

Grille de correction sociolinguistique extraite du CECRL.

On pourrait discuter et remettre en question les différents éléments ici présentés et censés déterminer les stades de développement de la compétence. Cependant, une telle grille a le mérite d'exister. Concentrons-nous sur les derniers niveaux puisqu'ils représentent le but à atteindre : ils donnent les critères qui permettent d'évaluer la réussite ou non du parcours d'apprentissage. En l'occurrence, ce qui est attendu aux niveaux $\mathrm{C} 1$ et $\mathrm{C} 2$ met en avant la maitrise de différentes formes d'une langue, y compris celles que la tradition scolaire déconsidère en LM. Une fois encore, on éclaire la contradiction des logiques puisque la «maitrise de la langue» (LM) vise, au contraire, la réduction des compétences langagières aux seules formes relevant de ce qui est présenté comme le registre de langue courant/soutenu : il n'existe aucun équivalent de la grille de «correction sociolinguistique». Plutôt que d'accompagner les élèves dans la gestion des différentes formes du français qu'ils vont acquérir au fil de leurs expériences langagières, parmi lesquelles la forme légitime, les enseignements se déroulent comme si les formes «collatérales» pouvaient être éradiquées, écrasées par le caractère absolument «bon» de l'usage enseigné. 


\section{La «langue maternelle» comme instrument de l'idéologie du standard}

Cette approche de la variation s'inscrit dans un cadre idéologique qui s'impose bien au-delà des murs de l'école. Il est de tradition en France d'ignorer la variation stylistique dans les discours prescriptifs en lui substituant une représentation de l'usage binaire (bon ou mauvais), pour mieux servir la tendance à un idéal unilinguisme ${ }^{10}$. On peut ainsi interdire des formes, les dévaloriser, les bannir, le ton étant donné par l'institution mère : on trouve sur le site internet de l'Académie à l'adresse des usagers du français de nombreuses rubriques où l'on propose explicitement des règles d'usage de la langue qui ne font aucun doute quant à l'absence de prise en compte de la variation, comme c'est le cas ici, dans la rubrique «Exemples de remarques normatives», au sujet de l'interrogation :

\section{INTERROGATION n. f. (...)}

L'interrogation directe exige l'inversion du sujet et du verbe. On doit dire : Quelle heure est-il ? et non Quelle heure il est ? Cette règle ne souffre aucune exception.

Extrait des «Exemples de remarques normatives» (<http://academie-francaise.fr/le-dictionnaire-la-ge-edition/exemplesde-remarques-normatives>, consulté le 19 avril 2013).

Il n'est même pas ici question de considérer qu'il pourrait y avoir d'autres formes d'interrogatives correspondant à des niveaux de langues «inférieurs », comme on le ferait dans un manuel scolaire. La variation y est interdite. En fait, à ce niveau institutionnel, il n'y a même pas de tentative de reconstruction d'une réalité qui, dans une manœuvre séductrice, pour mieux rallier à sa cause les locuteurs errants, peut laisser croire que ce qui circule effectivement entre les locuteurs est pris en compte. Il s'agit de légiférer, prescrire, voire imposer un usage unique, invalidant et excluant, du même coup, tous les autres. Cette conception de la langue raisonne dans la folk linguistique (Paveau, 2000) et le normativisme organise les représentations communes. En pratique,

10. «L'homogénéité prêtée à la langue est, avec l'unilinguisme, l'un des piliers essentiels de "l'idéologie du standard", très vivace dans l'histoire du français, et très présente dans sa diffusion, que ce soit dans la francophonie ou dans l'enseignement du français comme langue étrangère. » (Gadet, 2004, p. 19) 
les acteurs de terrain, les enseignants, les formateurs d'enseignants, les outils pédagogiques, ont leur part de responsabilité. Il peut s'agir d'atténuer la radicalité institutionnelle, en proposant des stratégies de contournement, et, inversement, être à l'origine d'un retour à davantage de rigidité, quitte à faire un pas hors du CECRL. Auger (2010) en constate les effets auprès d'élèves allophones nouvellement arrivés (EANA) en France : «Finalement, quand le français devient la langue à atteindre, les paramètres sociolinguistiques et didactiques sont brouillés par les représentations. » (p. 86) L'auteur montre comment le cadre dans lequel sont accueillis les EANA n'est pas propice au respect des principes de l'interculturalité. En s'inscrivant dans le cadre scolaire, dans l'objectif de créer un pont vers l'enseignement du français LM, l'enseignement du français, bien que langue étrangère, n'est pas abordé comme l'enseignement d'une langue vivante. Ceci est d'autant plus problématique que les EANA, plus que n'importe quels autres apprenants de français, sont complètement immergés dans le bain des pratiques langagières de la communauté française. Si un étudiant chinois, ayant appris le français en Chine, vient en France pour parfaire son apprentissage à l'université, il aura sans doute bien moins d'occasions de constater le décalage entre les enseignements et les usages réels qu'un enfant dont les parents sont installés en France. Pourtant, si, dans les deux cas, le français est une langue à acquérir, le discours à l'adresse des EANA est beaucoup plus proche de celui portant sur le français $\mathrm{LM}^{11}$.

Par ailleurs, d'autres études montrent qu'en dépit de ce qui a pu être dit précédemment sur l'enseignement du FLE, sur le terrain, les choses ne sont pas aussi idéales qu'attendues ${ }^{12}$. Il semble qu'on ait beaucoup de mal à adopter le CECRL quand il s'agit d'enseigner le français en France.

Ainsi, du point de vue de l'enseignement (et sans doute, dans une certaine mesure, de celui des représentations communes) il y aurait

11. S'il est souvent question, dans un premier temps, d'utiliser des méthodes de FLE pour enseigner le français aux EANA, l'objectif est de les amener le plus rapidement possible à intégrer le circuit «normal», c'est-à-dire l'intégration aux cours de français LM. Cela oriente le travail des enseignants et la part du travail consacrée à la variation et aux compétences pragmatique et sociolinguistique constatée dans le cadre du FLE (comme pour toutes les langues vivantes étrangères) est de fait réduite.

12. Par exemple Modard (2010), à partir d'un corpus de manuels FLE, montre l'absence de prise en compte de la variation. 
une gradation dans la conception de ce qu'est une langue vivante : le FLE occuperait une position intermédiaire entre le français langue LM et une autre langue étrangère. L'enseignement du FLE se nourrit de tout ce qui peut être dit de l'enseignement des langues vivantes, mais il semble que l'idéologie conservatrice qui imprègne les représentations reprend malgré tout parfois ses droits.

S'ils [les manuels de FLE] sont d'origine hexagonale, l'obligation d'être politiquement corrects les fait certes s'ouvrir à la diversité culturelle et géographique de la francophonie, la diversité linguistique de l'espace francophone est toutefois très souvent masquée par les belles images de plages martiniquaises ou du château Frontenac enneigé. Les auteurs des manuels dans les pays non francophones font preuve de plus d'ouverture, mais la variation diatopique n'est introduite qu'à des doses homéopathiques, à moins qu'elle ne fasse l'objet d'un traitement totalement artificiel, comme nous avons pu le démontrer ailleurs (cf. Pöll, 2000). (Pöll, 2005, p. 15)

Après tous ces constats, peut-on encore affirmer que le français est une langue vivante? Non, si l'expression s'ancre dans la problématique de l'enseignement des langues. L'emploi de «langue vivante», comme on a pu le voir, implique l'acceptation du caractère variable, donc insaisissable, de l'objet. Dans les discours qui encadrent l'enseignement, ceci vaut tant que l'objet en question n'est pas la LM. Pourtant, le développement du répertoire langagier des élèves s'accompagne de davantage d'ouverture d'esprit, d'ouverture à la diversité. C'est ce qu'on peut effectivement lire dans les textes officiels, à propos de l'enseignement des langues étrangères à l'école :

L'apprentissage d'une langue étrangère développe la sensibilité aux différences et à la diversité culturelle. Il favorise [...] l'ouverture d'esprit et la compréhension d'autres façons de penser et d'agir.

En revanche, l'enseignement de la LM n'a pas pour objectif «la sensibilité aux différences ». Au contraire, le français LM est figé dans un état : son enseignement vise l'uniformisation des compétences langagières. Dans ce contexte, les élèves français suivent un cursus qui devrait les amener à revoir leur répertoire langagier : certaines entrées n'y ont pas leur place, celles qui, selon la catégorisation scolaire des formes, illustrent le bas de l'échelle des «niveaux de langue». Évidemment, les choses ne sont pas aussi clairement énoncées dans les textes présentant le socle commun de connaissances et de compétences. On peut par exemple y lire : 
L'intérêt pour la langue comme instrument de pensée et d'insertion développe :

- la volonté de justesse dans l'expression écrite et orale, du goût pour l'enrichissement du vocabulaire;

- le goût pour les sonorités, les jeux de sens, la puissance émotive de la langue;

- l'intérêt pour la lecture (des livres, de la presse écrite);

- l'ouverture à la communication, au dialogue, au débat ${ }^{13}$.

Ce qu'on ne dit pas ici, mais que l'on comprend en observant les programmes, les manuels et, par extension, les pratiques de classe, c'est que relève de la «justesse» ce qui est conforme au modèle attendu. Il ne s'agit pas de penser la justesse relativement au repérage de contraintes stylistiques. Par exemple, l' «enrichissement du vocabulaire» ne vise pas l'accumulation d'items afin d'assurer la variation stylistique. Il s'agit, au contraire, d'en éradiquer certains pour y installer ceux présentés comme légitimes. Par contraste avec ce qui est dit à propos de l'enseignement des langues vivantes (étrangères), on est en droit de s'interroger sur les valeurs humaines transmises : si la prise en compte de la variation, la «sensibilité aux différences», favorise «l'ouverture d'esprit et la compréhension d'autres façons de penser et d'agir», alors l'inverse doit aussi être vrai.

\section{Conclusion}

Au-delà des discussions sur l'aspect terminologique du problème posé, on voit (du moins, j'ai tenté de montrer) que l'enseignement scolaire de la langue dite maternelle est contraint par une appréhension de la langue et de ses usages conditionnée par la nécessaire reconnaissance d'un modèle unique et communément partagé. Dès lors, on pourrait être tenté d'exclure de la réflexion didactique la variation (stylistique), considérant que la compétence sociolinguistique et pragmatique relève d'apprentissages extrascolaires. Cependant, on voit mal comment tenir un discours sur la langue qui ne prendrait pas en considération ce qui apparait comme une caractéristique fondamentale des usages langagiers.

13. Le socle commun de connaissances et de compétences. Tout ce qu'il est indispensable de maîtriser à la fin de la scolarité obligatoire, décret du 11 juillet 2006. 
Néanmoins, on peut se demander si une telle pratique, aussi absurde soit-elle, ne serait pas préférable à celle qui consiste à poser comme incontestablement «justes» et évidents des usages qui ne font pas écho à ce qui est appréhendable par les élèves, en dehors de l'école.

Loin de remettre en cause l'intérêt et la nécessité sociale de la diffusion d'un modèle standard, cet article invite à se questionner sur la façon dont il doit être abordé, sur les moyens d'accompagner les élèves à se représenter le modèle comme intégrant le champ des possibles de la langue, sans prendre un caractère exclusif et excluant. Il ne s'agit pas de nier la spécificité, la légitimité, le prestige du modèle standard, compte tenu de son rôle social. Les effets seraient contre-productifs dans l'entreprise de socialisation assignée à l'école. En revanche, il me semble que le discours scolaire gagnerait en cohérence et en efficacité en distinguant ce qui relève du linguistique et des jeux de valeur symbolique autour desquels s'articulent les représentations communes et s'organisent les interactions sociales.

Ainsi, on déconstruirait les tenants de l'opposition «langue maternelle» / «langue vivante» qui, bien qu'elle constitue le cœur de la réflexion présentée ici, n'a, en fait, aucun lieu d'être : si une langue est pratiquée et transmise (langue maternelle), elle est indéniablement vivante.

\section{RÉFÉRENCES BIBLIOGRAPHIQUES}

Andersen, Elaine Slosberg. (1990). Speaking With Style: The Sociolinguistic Skills of Children. Londres : Routledge.

Auger, Nathalie. (2010). Le français, cette autre langue à comparer une question en miroir : l'apprentissage et l'enseignement du français aux élèves nouvellement arrivés en France (ENA). Dans C. Corblin \& J. Sauvage (dir.), L'enseignement des langues vivantes étrangères à l'école. Impacts sur le développement de la langue maternelle (p. 79-96). Paris, France : L'harmattan.

BILliEZ, Jacqueline. (2007). Etre plurilingue, handicap ou atout. Les mots de l'immigration, 111, 88-90.

Bouillaud, Céline et coll. (2007). Cyberlangage et orthographe : quels effets sur le niveau orthographique des élèves de $\mathrm{CM} 2,5^{\mathrm{e}}, 3^{\mathrm{e}}$ ? Bulletin de psychologie, 492, 553-565.

Boulton, Alex (dir.). (2009). Des documents authentiques oraux aux corpus : questions d'apprentissage en didactique des langues, Mélanges CRAPEL, 31 . 
BOUTEYRE, Évelyne. (2004). Réussite et résilience scolaires chez les enfants de migrants. Paris, France : Dunod.

Charmeux, Évelyne. (1989). Le «bon français» et les autres. Toulouse : Éd. Milan.

Chiss, Jean-Louis \& DAVID, Jacques. (2012). Didactique du français et étude de la langue. Paris : Armand Colin.

David, Jacques \& Goncalves, Harmonie. (2007). L'écriture électronique, une menace pour la maitrise de la langue? Le français aujourd'hui, $156,39-47$.

Fairon, Cédric \& Klein, Jean-René. (2010). Les écritures et graphies inventives des SMS face aux graphies normées. Le français aujourd'hui, 170, 133-122.

GADET, Françoise. (2003). La variation sociale en français. Paris : Ophrys. GADET, Françoise. (2004). Quelle place pour la variation dans l'enseignement du français langue étrangère ou seconde? Prétextes franco-danois, 4, 17-28.

GADEt, Françoise. (2008). L'oral et l'écrit dans les changements technologiques et idéologiques. Dans E. Galazzi \& C. Molinari (dir.), Les français en émergence. Bern : Peter Lang.

GADET, Françoise \& Tyne, Henry. (2007). Le style en sociolinguistique : ce que nous apprend l'acquisition. Pratiques, 135-136, 91-99.

Genouvrier, Émile \& Gueunier, Nicole. (1982). Langue maternelle et communauté linguistique. Langue française, 54, 3-6.

GuERIN, Emmanuelle. (2008). Le 'français standard': une variété située? Dans J. Durand, B. Habert \& B. Laks (dir.), Actes du $1^{e r}$ congrès mondial de linguistique française (CMLF 08). Paris, France : Institut de linguistique française.

Guerin, Emmanuelle. (2013). La validité de la notion de «handicap linguistique» en question. Le français aujourd'hui, 183, 91-104.

Halliday, Michael Alexander Kirkwood. (2007), Language and Education (vol. 9). Londres : Continuum.

Heller, Monica. (1988). Strategic Ambiguity: Code-Switching in the Management of Conflict. Dans M. Heller (dir.), Code-Switching: Anthropological and Sociolinguistic Perspectives (p. 77-97). Berlin, Allemagne : Mouton de Gruyter.

Henri-Panabière, Gaëlle. (2010). Des «héritiers » en échec scolaire. Paris, France : La Dispute.

Herlitz, Wolfgang, Ongstad, Sigmund \& Van de Ven, Piet-Hein. (2007). Research on Mother Tongue Education in a Comparative International Perspective Theoretical and Methodological Issues. Utrecht, Pays-Bas : Rodopi. 
LAHIRE, Bernard. (1993). Culture écrite et inégalités scolaires. Lyon, France : Presses universitaires de Lyon.

LEDEgen, Gudrun. (2000). Le bon français. Les étudiants et la norme linguistique. Paris : L'Harmattan.

Milroy, James \& Milroy, Lesley. (1985). Authority in Language. Londres et New York : Routledge.

ModARD, Daniel. (2010). Familiariser des jeunes apprenants avec la diversité linguistique et culturelle d'une langue : le français d'ici et d'ailleurs. Dans C. Corblin \& J. Sauvage (dir.), L'enseignement des langues vivantes étrangères à l'école. Impacts sur le développement de la langue maternelle (p. 55-74). Paris : L'harmattan.

MougEon, Françoise. (1998). Variation stylistique en français et compétence communicative en milieu minoritaire. Dans A. Boudreau \& L. Dubois (dir.), Le français, langue maternelle, dans les collèges et les universités en milieu minoritaire (p. 63-88). Moncton, Canada : Éditions d'Acadie.

Paveau, Marie-Anne. (2000). La «richesse lexicale», entre apprentissage et acculturation. Le français aujourd'hui, 131, 19-30.

Paveau, Marie-Anne. (2008). La langue sans classes de la grammaire scolaire. Le français aujourd'hui, 162, 29-40.

Philonenko, Alexis. (2007). Langue morte et langue vivante. Revue de métaphysique et de morale, 54, 157-178.

PöLl, Bernhard. (2005). Le français langue pluricentrique? Études sur la variation diatopique d'une langue standard. Francfort-sur-le-Main, Allemagne : PeterLang.

VIGNeR, Gérard. (2003). Nommer le français. Revue de didactologie des langues-cultures, 130, 153-166. 